\title{
Reconstructing a 3D Line from a Single Catadioptric Image
}

\author{
Douglas Lanman* Megan Wachs ${ }^{\dagger}$ Gabriel Taubin* Fernando Cukierman ${ }^{\ddagger}$ \\ *Brown University, Providence, Rhode Island, USA \\ ${ }^{\dagger}$ Stanford University, Stanford, California, USA \\ $\ddagger$ University of Buenos Aires, Buenos Aires, Argentina
}

\begin{abstract}
This paper demonstrates that, for axial non-central optical systems, the equation of a $3 D$ line can be estimated using only four points extracted from a single image of the line. This result, which is a direct consequence of the lack of vantage point, follows from a classic result in enumerative geometry: there are exactly two lines in 3-space which intersect four given lines in general position. We present a simple algorithm to reconstruct the equation of a $3 D$ line from four image points. This algorithm is based on computing the Singular Value Decomposition (SVD) of the matrix of Plücker coordinates of the four corresponding rays. We evaluate the conditions for which the reconstruction fails, such as when the four rays are nearly coplanar. Preliminary experimental results using a spherical catadioptric camera are presented. We conclude by discussing the limitations imposed by poor calibration and numerical errors on the proposed reconstruction algorithm.
\end{abstract}

\section{Introduction}

In this paper we study the process of image formation in axial non-central optical systems. While lacking a unique vantage point, these systems possess an axis of symmetry which every $3 \mathrm{D}$ ray associated with an image pixel intersects. One such system is a catadioptric camera composed of a pinhole camera and a convex spherical mirror. Typical images captured with such a system are shown in Figure 1. Even though the implementation presented in this paper uses a spherical catadioptric system, the analytic results and algorithm apply to any axial non-central catadioptric system for which the mapping from pixel coordinates to rays in $3 \mathrm{D}$ is known.

The simplest model for image formation is the pinhole model. In its most abstract form, it consists of an image plane where images are formed and an external point called the pinhole. The image of a point in space is defined by the intersection of the line determined by the 3D point and the pinhole with the image plane. A straight line in 3D,

\footnotetext{
* \{douglas_lanman, taubin $\} @$ brown.edu
}
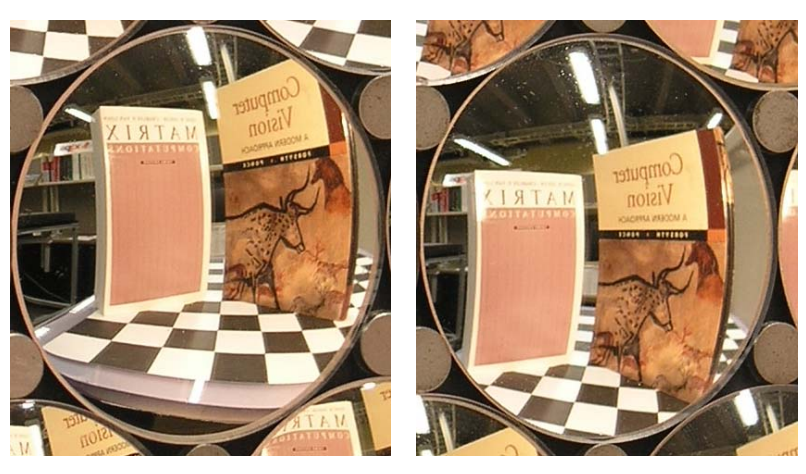

Figure 1: Images formed by a spherical catadioptric camera.

not containing the pinhole, projects onto a straight line contained in the image plane called the image line. The straight line in 3D and the pinhole span a plane. The intersection of this plane with the image plane is the image line. Note that two points on the image line determine the plane containing the 3D line, but the 3D line cannot be uniquely determined from the information provided by the two image points. Two degrees of freedom remain undetermined and, as a result, more points on the image line do not provide any additional information.

A stereo algorithm, which uses two images taken from different viewpoints, is required to fully reconstruct a $3 \mathrm{D}$ line. In addition, a stereo algorithm requires establishing correspondence between the two image lines, which usually is a time-consuming and error-prone process, even when done manually. In contrast with this situation, we show in this paper that an axial non-central catadioptric system can fully reconstruct a 3D line from a single image without the need to establish correspondences. This is a direct consequence of the lack of a single vantage point. However, there are some intrinsic limitations due to rays in singular configurations and numerical conditioning issues. These problems correspond to the poor reconstruction results obtained in short baseline stereo systems. We analyze these cases and present simulations to determine the minimum acceptable calibration tolerances to produce reliable reconstructions. 


\section{Related Work}

Swaminiathan et al. [20] study catadioptric cameras without a unique vantage point. These authors describe the benefits of multiple-viewpoint systems, where the multiple viewpoints are described by a caustic surface. Nayar [14] describes a stereo reconstruction algorithm using two reflective spheres and an orthographic camera. Nene and $\mathrm{Na}-$ yar [15] describe four catadioptric systems with planar, ellipsoidal, hyperboloidal, and paraboloidal mirrors. They use two mirrors to do depth mapping and 3D reconstruction. Geyer and Daniilidis [6] use a paraboloid mirror and an orthographic lens. They show that projections of two sets of parallel lines suffice for intrinsic calibration. Kang [11] studies a catadioptric camera with a parabolic mirror to capture video sequences. Geyer and Daniilidis [7] describe a unifying model for central catadioptric cameras (with a single unifying viewpoint). Geyer and Daniilidis [8] again use a parabolic catadioptric device and study the geometry of two uncalibrated views. Here Euclidean reconstruction from two views is possible. Barreto and Araujo [1] study catadioptric projection of lines in central catadioptric systems (with a single effective viewpoint). Ying and $\mathrm{Hu}$ [22] use the projections of spheres with an orthographicparabolic system to calibrate a catadioptric system (as opposed to the projections of lines). Hicks and Perline [10] design catadioptric sensors for rectifying images. Finally, in a closely related paper, Caglioti [3] also addresses the problem of reconstructing a 3D line from one image in an axialsymmetric catadioptric system, and presents an algorithm, analysis, and experimental results restricted to a catadioptric camera with a conical mirror. In this paper we present a more general approach for 3D line reconstruction in axial non-central optical systems.

\section{Spherical Catadioptric Ray Equations}

In this section we review the analytic mapping from image points to $3 \mathrm{D}$ rays for a spherical catadioptric system. Our analysis and line reconstruction algorithm are valid, however, for more general cameras $[9,19]$.

The reflection of rays on the spherical mirror is governed by the classical law of reflection: the incident ray, the normal vector to the mirror surface at the point of incidence, and the reflected ray are coplanar. In addition, the incident and reflected rays are symmetric with respect to the normal vector, as shown in Figure 2. This means that the optical system has cylindrical symmetry with respect to the line joining the pinhole and the center of the spherical mirror. We call this line the optical axis of the system.

To analyze the process of image formation in this system we consider rays traveling in the opposite direction, from the camera through the pinhole to space, after they are re-

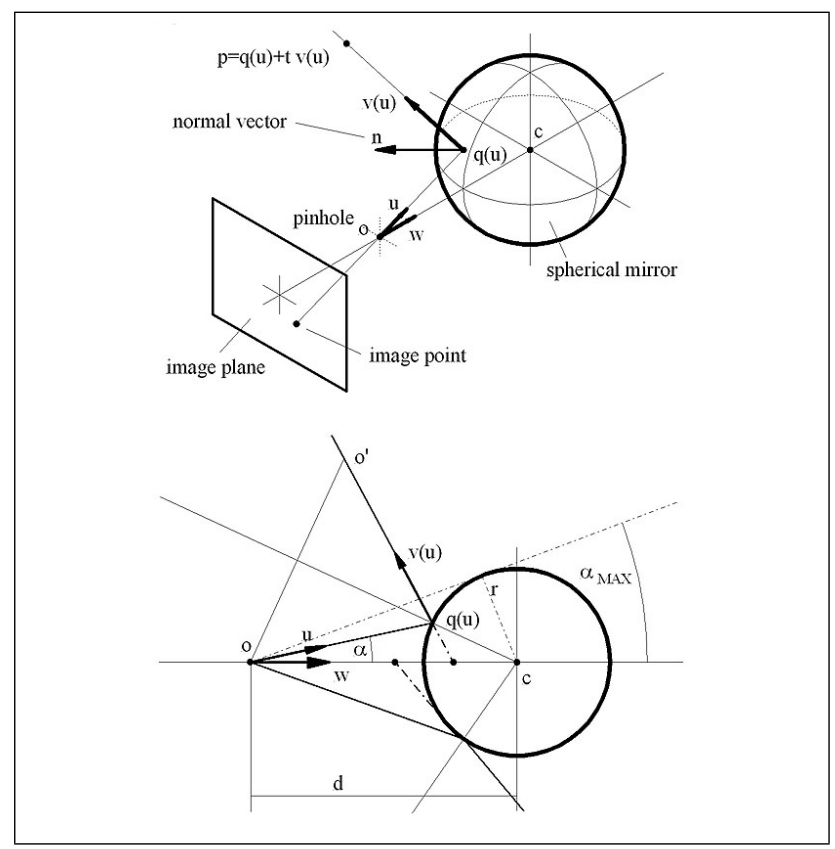

Figure 2: The geometry of a reflected ray. The incident ray, normal vector, and reflected ray are coplanar.

flected off of the spherical mirror, as illustrated in Figure 2(a). Here we denote $o$ the pinhole, $c$ the center of the spherical mirror, $d$ the distance between the pinhole and the center of the sphere, $r$ the radius of the spherical mirror, and $w$ the vector from $o$ to $c$ normalized to unit length, so that $c-o=d w$. Instead of an image plane, we use a spherical retina. Image points are unit-length vectors $u$. The origin of the world coordinate system is placed at the pinhole.

Because of the coplanarity of incident ray, reflected ray, and optical axis, the reflected ray intersects the optical axis. But not all these rays intersect the axis at the same point. This is illustrated in Figure 2(b). If two image points $u_{1}$ and $u_{2}$ are not congruent modulo a rotation around the optical axis, the corresponding reflected rays are twisted, i.e., the two supporting lines are not coplanar. This property makes the reconstruction of a $3 \mathrm{D}$ line from a single image possible.

Let $u$ be an arbitrary unit length vector and let

$$
I_{u}=\{q=\xi u: \xi>0\}
$$

be the incident ray that goes through the pinhole in the direction of $u$. It hits the mirror surface at the point $q=q(u)$, and reflects off according to the reflection law along a vector $v=v(u)$. The reflected ray $R_{u}$ can be written in parametric form as

$$
R_{u}=\{p=q(u)+t v(u): t \geq 0\},
$$

where $v=v(u)$ is its direction. 
For a spherical mirror we have

$$
q(u)=\left(d\left(u^{t} w\right)-\sqrt{r^{2}-d^{2}\left(1-\left(u^{t} w\right)^{2}\right)}\right) u .
$$

and we define the vector $v$ as the mirror image of the incident vector $u$ with respect to tangent plane to the mirror surface at $q$ [14]:

$$
v(u)=\left(I-2 n n^{t}\right) u,
$$

where $n=n(u)=(q(u)-c) / r$. A detailed derivation is presented in [13].

\section{The Image of a 3D Line}

We determine in this section the implicit equation of the image of a 3D line $L$. This is an implicit equation in a unit length pixel vector $u$. This derivation is valid not only for spherical catadioptric cameras, but for general cameras [9] [19] where the mapping from pixels to rays is available.

We first take care of some special cases. Let $L_{0}$ be the optical axis of the system, and let $L$ be another 3D line. If $L$ contains a reflected ray the projection of $L$ is a single point. The converse of this fact is also true. If $L$ intersects $L_{0}$ or if $L$ is parallel to $L_{0}$, there is a plane containing $L$ and $L_{0}$. In this case, since the projection is contained in the same plane, it must be contained in the straight line defined by the containing plane and the image plane. This line contains the image of the center of the spherical mirror.

The general case is when $L$ and $L_{0}$ are twisted, i.e., when they are not coplanar. In this case the image of $L$ is not a straight line. In this case, for every point $p$ in $L$ there is a unique vector $u$ and $t>0$ such that $p=q(u)+t v(u)$. For this particular value of $u$, the ray $R_{u}$ intersects the line $L$, and the intersection point is $p$. The implicit equation of the image of $L$ is a function of $u$ which is zero if and only if the ray $R_{u}$ intersects the line $L$.

$$
\left\{u: R_{u} \cap L \neq \emptyset\right\}=\left\{u: \phi_{L}(u)=0\right\},
$$

where $\phi_{L}$ includes the intrinsic calibration parameters.

To get an explicit expression for $\phi_{L}$, it is better to work in projective space. A point in 3-dimensional projective space $\mathbb{P}^{3}$ is represented by its homogeneous coordinates $x=\left(x_{1}, x_{2}, x_{3}, x_{4}\right)^{t}$. The homogeneous coordinates of a finite three-dimensional point $p$ are $\left(p_{1}, p_{2}, p_{3}, 1\right)^{t}$, which we also denote $p$. A line in $\mathbb{P}^{3}$ is represented by a $4 \times 2$ matrix $L$ of rank 2 (we use the same symbol for the line and its representing matrix) so that a point $x$ belongs to $L$ if and only if $L^{t} x=0$. This representation is not unique: two matrices $L$ and $K$ represent the same line if $L=K A$ for some non-singular $2 \times 2$ matrix $A$. This is an equivalence relation in the space of $4 \times 2$ rank 2 matrices. The Grassman manifold $G(1,3)$ is the space of all the lines in $\mathbb{P}^{3}$, i.e., the space of all the $4 \times 2$ rank 2 matrices modulo this equivalence.
To find the implicit equation we replace the parametric representation of $R_{u}$ in the implicit equations of $L$ and eliminate the variable $t$. Let

$$
L=\left(\begin{array}{ll}
l_{11} & l_{21} \\
l_{12} & l_{22} \\
l_{13} & l_{23} \\
l_{14} & l_{24}
\end{array}\right)
$$

be the matrix defining the line $L$ in implicit form. Replacing the parametric representation of $p$ in the implicit equations of $L$ we obtain

$$
\left\{\begin{array}{l}
l_{1}^{t}(t v+q)+l_{14}=0 \\
l_{2}^{t}(t v+q)+l_{24}=0
\end{array}\right.
$$

where $l_{1}=\left(l_{11}, l_{12}, l_{13}\right)^{t}$ and $l_{2}=\left(l_{21}, l_{22}, l_{23}\right)^{t}$. Then we eliminate $t$ from these two equations and obtain

$$
\left(\left(l_{1}^{t} v\right)\left(l_{2}^{t} q\right)-\left(l_{2}^{t} v\right)\left(l_{1}^{t} q\right)\right)+\left(\left(l_{1}^{t} v\right) l_{24}-\left(l_{2}^{t} v\right) l_{14}\right)=0
$$

which can be written in matrix form as follows

$$
\left(\begin{array}{c}
v_{1} \\
v_{2} \\
v_{3}
\end{array}\right)^{t}\left(\begin{array}{cccc}
0 & L_{12} & L_{13} & L_{14} \\
-L_{12} & 0 & L_{23} & L_{24} \\
-L_{13} & -L_{23} & 0 & L_{34}
\end{array}\right)\left(\begin{array}{c}
q_{1} \\
q_{2} \\
q_{2} \\
1
\end{array}\right)=0
$$

where $L_{i j}$ is the determinant of the $2 \times 2$ minor of $L$ composed of the $i$-th and $j$-th rows. For example, $L_{23}=$ $l_{12} l_{23}-l_{22} l_{13}$. The elements of the vector

$$
\left(L_{12}, L_{13}, L_{14}, L_{23}, L_{24}, L_{34}\right)^{t}
$$

are the Plücker coordinates of $L$. They define an injection $G(1,3) \rightarrow \mathbb{P}^{5}$ [12]. It is easy to see that this mapping is well defined: if $L$ and $K$ are two representations of the same line, and $L=K A$ for a $2 \times 2$ matrix $A$, the Plücker coordinates of $L$ are equal to the Plücker coordinates of $K$ multiplied by the determinant of $A$.

Note that the expression in Equation 5 is linear and homogeneous in the Plücker coordinates of $L$, and can be expressed as an inner product

$$
\phi_{L}(u)=\left(\begin{array}{c}
v_{1} q_{2}-v_{2} q_{1} \\
v_{1} q_{3}-v_{3} q_{1} \\
v_{1} \\
v_{2} q_{3}-v_{3} q_{2} \\
v_{2} \\
v_{3}
\end{array}\right)^{t}\left(\begin{array}{c}
L_{12} \\
L_{13} \\
L_{14} \\
L_{23} \\
L_{24} \\
L_{34}
\end{array}\right),
$$

where the left hand side six-dimensional vector whose coordinates are functions of $v$ and $q$ is the vector of Plücker coordinates of the matrix

$$
\left(\begin{array}{cc}
v_{1} & q_{1} \\
v_{2} & q_{2} \\
v_{3} & q_{2} \\
0 & 1
\end{array}\right)
$$


which we will also denote $R_{u}$. Finally,

$$
\phi_{L}(u)=R_{u}^{t} L .
$$

We have shown here that the necessary and sufficient condition for a first line $R_{u}$ defined in parametric form and a second line $L$ defined in implicit form to intersect is that the inner product of their corresponding vectors of Plücker coordinates be equal to zero.

\section{Two Lines Intersect Four Given Lines}

In 1874 Schubert published a celebrated treatise on Enumerative Geometry [16] which dealt with finding the number of points, lines, planes, etc., satisfying certain geometric conditions. These were important problems in Schubert's time. Several authors have explained Schubert calculus in contemporary language $[5,12]$. One of the simplest problems in this field is: how many lines in 3-space, in general, intersect four given lines? The answer is two. It has been shown that this result has applications in computational geometry $[17,4,18]$ and computer graphics [21]. As shown in Section 7, there is an infinite number of lines intersecting three or less lines, and in general none intersecting more than four lines. This is so unless the lines are arranged in a special configuration, such as all the reflected rays corresponding to a pixel in the image of a $3 \mathrm{D}$ line.

Although algorithms to find the two lines which intersect four given lines have been published [21], here we present a variant which operates on given lines in parametric form and intersecting lines in implicit form, well-adapted to our problem.

We have introduced Plücker coordinates in the previous section. The 6-dimensional vector $R_{u}$ of Equation 7 defines a hyperplane in $\mathbb{P}^{5}$, namely $H_{u}=\left\{X: R_{u}^{t} X=0\right\}$, where $\left(X_{12}, X_{13}, X_{14}, X_{23}, X_{24}, X_{34}\right)^{t}$ are the homogeneous coordinates of a point $X$ in $\mathbb{P}^{5}$. The set of lines $L$ in $\mathbb{P}^{3}$ which intersect a given ray $R_{u}$ belong to the hyperplane $H_{u}$ in $\mathbb{P}^{5}$.

Not every point in $\mathbb{P}^{5}$ is the Plücker coordinate of a line in $\mathbb{P}^{3}$ though. Actually the image of the Plücker map $G(1,3) \rightarrow \mathbb{P}^{5}$ forms a quadric $Q$ in $\mathbb{P}^{5}[12]$ defined by the zeros of the polynomial

$$
f(X)=X_{12} X_{34}-X_{13} X_{24}+X_{14} X_{23} .
$$

Given four different rays $R_{u_{1}}, R_{u_{2}}, R_{u_{3}}$, and $R_{u_{4}}$ in $\mathbb{P}^{3}$ in general position, the intersection of their corresponding hyperplanes in $\mathbb{P}^{5}$ is a line. In general, the intersection of this line with the quadric $Q$ consists of two points. The two lines whose Plücker coordinates are these two points in $\mathbb{P}^{5}$ are the two lines which intersect the original four.

To compute the two intersection points in $\mathbb{P}^{5}$ we form a $6 \times 4$ matrix concatenating the Plücker coordinates of the four lines as columns $R=\left[R_{u_{1}}\left|R_{u_{2}}\right| R_{u_{3}} \mid R_{u_{4}}\right]$. The line in $\mathbb{P}^{5}$ which we are looking for can be described in implicit form as the set of points $X$ satisfying $R^{t} X=0$. This line can also be defined in parametric form as $X=$ $X_{1}+t X_{2}, X_{1}$ and $X_{2}$ are two different points satisfying $R^{t} X_{1}=R^{t} X_{2}=0$, and $t$ is a parameter. We use the SVD algorithm to solve this problem. $X_{1}$ and $X_{2}$ are two left singular vectors of $R$ associated with the two smallest singular values, which in this case are both zero. They span the subspace orthogonal to the columns of $R$, but since they are both associated with the same singular value, they are not unique, and we can replace what the SVD algorithm computes with any pair of orthonormal vectors spanning the same subspace. In particular, since the vector of Plücker coordinates of the optical axis $L_{0}$ represented in implicit form belongs to this subspace, we can take $X_{1}=L_{0}$, and $X_{2}$ any orthogonal unit length vector in the two-dimensional subspace associated with the singular value zero. For example, of the two smallest singular vectors of $R$, pick the most orthogonal to $L_{0}$. Finally, we need to find the two roots of the equation

$$
f\left(X_{1}+t X_{2}\right)=0
$$

but based on what we just discussed, one of these roots is $t=0$, and the problem reduces to finding one root of a linear equation in one variable. For this we expand the previous expression

$$
f\left(X_{1}+t X_{2}\right)=f\left(X_{1}\right)+t b\left(X_{1}, X_{2}\right)+t^{2} f\left(X_{2}\right)
$$

where $b(X, Y)$ is the bilinear form

$X_{12} Y_{34}-X_{13} Y_{24}+X_{14} Y_{23}+X_{23} Y_{14}-X_{24} Y_{13}+X_{34} Y_{12}$.

For the expression above to have two different roots, we need $f\left(X_{2}\right) \neq 0$ and $b\left(X_{1}, X_{2}\right) \neq 0$. Since $f\left(X_{1}\right)=0$ the non-zero root is

$$
t=-b\left(X_{1}, X_{2}\right) / q\left(X_{2}\right),
$$

and the Plücker coordinates of the intersecting line that we were looking for is

$$
X=f\left(X_{2}\right) X_{1}-b\left(X_{1}, X_{2}\right) X_{2} .
$$

We finish this section by computing the vector $X_{1}=L_{0}$ of Plücker coordinates of the optical ray represented in implicit form. We first choose two vectors $w^{1}$ and $w^{2}$ orthogonal to $w$ so that $\left\{w^{1}, w^{2}, w\right\}$ form an orthonormal frame, and so $w=w^{1} \times w^{2}$. Since the optical ray contains the origin, the following matrix represents it in implicit form

$$
\left(\begin{array}{cc}
w_{1}^{1} & w_{1}^{2} \\
w_{2}^{1} & w_{2}^{2} \\
w_{3}^{1} & w_{3}^{2} \\
0 & 0
\end{array}\right) .
$$


The Plücker coordinates of this matrix are

$$
\left(\begin{array}{c}
w_{1}^{1} w_{2}^{2}-w_{2}^{1} w_{1}^{2} \\
w_{1}^{1} w_{3}^{2}-w_{3}^{1} w_{1}^{2} \\
0 \\
w_{2}^{1} w_{3}^{2}-w_{3}^{1} w_{2}^{2} \\
0 \\
0
\end{array}\right)=\left(\begin{array}{c}
\left(w^{1} \times w^{2}\right)_{3} \\
-\left(w^{1} \times w^{2}\right)_{2} \\
0 \\
\left(w^{1} \times w^{2}\right)_{1} \\
0 \\
0
\end{array}\right)=\left(\begin{array}{c}
w_{3} \\
-w_{2} \\
0 \\
w_{1} \\
0 \\
0
\end{array}\right)
$$

independently of the choice of $w^{1}$ and $w^{2}$.

\section{Inverting Plücker Coordinates}

The last step in our algorithm is to invert the Plücker coordinates of the solution: given a point $X$ in the Plücker quadric $Q \subseteq \mathbb{P}^{5}$, find a $4 \times 2$ matrix $L$ of rank 2 so that its Plücker coordinates are equal to $X$ in $\mathbb{P}^{5}$, i.e., modulo a multiplicative factor. Since every line can be represented as the intersection of two orthogonal planes, one of which contains the pinhole $o$ (the coordinate system origin), we seek here a solution in implicit form where the matrix is written as

$$
L=\left(\begin{array}{cc}
n_{b 1} & n_{o 1} \\
n_{b 2} & n_{o 2} \\
n_{b 3} & n_{o 3} \\
-d & 0
\end{array}\right)
$$

$n_{o}$ and $n_{b}$ are two unit length orthogonal vectors, and $d=0$ if and only if the line contains the origin. If a parametric representation of the line is preferred, it is easy to verify that the same line can be described as the set of points $p(t)=$ $t n_{t}+p_{0}$, where $n_{t}=n_{b} \times n_{o}$ and $p_{0}=d n_{b}$. We have to solve the following system of equations for $n_{o}$ and $n_{c}$

$$
\begin{cases}n_{b 1} n_{o 2}-n_{b 2} n_{o 1} & =\nu X_{12} \\ n_{b 1} n_{o 3}-n_{b 3} n_{o 1} & =\nu X_{13} \\ d n_{o 1} & =\nu X_{14} \\ n_{b 2} n_{o 3}-n_{b 3} n_{o 2} & =\nu X_{23} \\ d n_{o 2} & =\nu X_{24} \\ d n_{o 3} & =\nu X_{34}\end{cases}
$$

where $\nu$ is a non-zero constant. Let $X_{o}=\left(X_{14}, X_{24}, X_{34}\right)^{t}$ and $X_{t}=\left(X_{23},-X_{13}, X_{12}\right)^{t}$. If $X_{o}=0$ we have $d=0$, and so, the line passes through the origin. In this case the solution is not unique. Since

$$
n_{t}=n_{b} \times n_{o}=\left(\begin{array}{c}
n_{b 2} n_{o 3}-n_{b 3} n_{o 2} \\
n_{b 3} n_{o 1}-n_{b 1} n_{o 3} \\
n_{b 1} n_{o 2}-n_{b 2} n_{o 1}
\end{array}\right)=\nu X_{t},
$$

we compute $n_{t}=X_{t} /\left\|X_{t}\right\|$, choose any unit length vector $n_{o}$ orthogonal to $n_{t}$, and set $n_{b}=n_{o} \times n_{t}$. If $X_{o} \neq 0$ we can compute $n_{o}=X_{o} /\left\|X_{o}\right\|, n_{t}=X_{t} /\left\|X_{t}\right\|$ and $n_{b}=$ $n_{o} \times n_{t}$. Note that $n_{o}$ and $n_{t}$ are orthogonal because $X$ belongs to the Plücker quadric

$$
d n_{o}^{t} n_{t}=\nu^{2} X_{o}^{t} X_{t}=f(X)=0 .
$$

To compute the remaining parameter $d$, we first compute $\nu$

$$
n_{t}=n_{b} \times n_{o}=\nu X_{t} \quad \Rightarrow \quad \nu=1 /\left(n_{t}^{t} X_{t}\right)
$$

and then $d$

$$
d n_{o}=\nu X_{o} \quad \Rightarrow \quad d=\left(n_{o}^{t} X_{o}\right) /\left(n_{t}^{t} X_{t}\right) .
$$

\section{Four Lines in General Position}

It is difficult to describe the necessary and sufficient geometric conditions in $\mathbb{P}^{3}$ for four rays to be in general position. In the end, what we want is the necessary and sufficient condition for the intersection of the four hyperplanes to be a line and for the line to intersect the Plücker quadric in two points, so that the steps described above result in exactly two different lines. This condition is that the matrix $R$ be full rank, i.e.,

$$
\operatorname{rank}\left[R_{u_{1}}\left|R_{u_{2}}\right| R_{u_{3}} \mid R_{u_{4}}\right]=4 .
$$

Since we test this condition during the computation, it is natural to take it as the definition of being in general position. This is justified: the sets of four lines not in general position satisfy some algebraic equations and are contained in a set of measure zero. Small perturbations of coefficients bring them to the general case.

There are some geometric configurations of four lines which result in singular positions. The case of the line $L$ intersecting the optical axis $L_{0}$ was mentioned above. In this case all the rays are contained in the plane defined by $L$ and $L_{0}$ and the image of the line is also a straight line (if imaged on an image plane). As in the case of a pinhole camera, a unique line cannot be determined.

However, there are non-coplanar configurations of four lines which are singular. Given three lines in general position, such as when they are pairwise twisted, there exists a unique ruled conic in 3D which contains the three lines. In the case of pairwise twisted lines, this conic is a hyperboloid of one sheet. The ruled conic containing the three lines also contains all the members of two one-parameter families of lines. Two lines of the same family do not intersect, and each member of one family intersects all the members of the other family. If in a set of four twisted lines the fourth line belongs to the ruled quadric defined by the first three, then $R$ must be rank-deficient, because there are an infinite number of lines intersecting the given four. If $\rho(p)$ is a polynomial in $p \in \mathbb{R}^{3}$, then $\rho(q+t v)$ is a univariate polynomial in $t$ of the same degree. For the set of zeros of $\rho$ to contain the line $\{p+t v\}$, it is necessary the polynomial in $t$ be identically zero. That is, all its coefficients must be zero. These conditions are homogeneous linear equations in the coefficients of $\rho(p)$. If $\rho(p)$ is quadratic, it has 10 coefficients, and we have 3 linear equations for each line. From 3 lines 


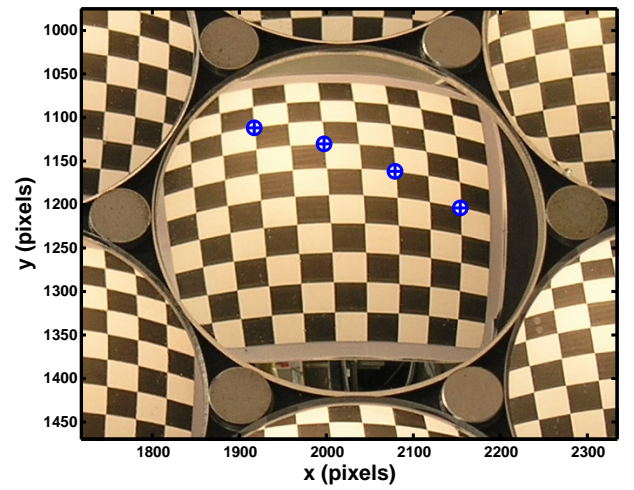

(a) Catadioptric image example. Points used for reconstruction are indicated by crosshairs.

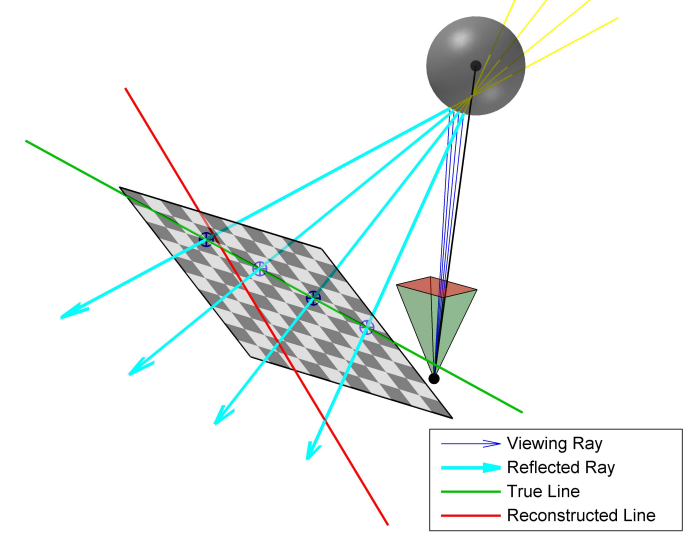

(b) $3 \mathrm{D}$ reconstruction of labeled points.

Figure 3: Single image reconstruction results. The mirror shown in (a) is represented by a gray sphere in (b). The checkerboard is placed at its optimized position.

we get 9 homogeneous linear equations in 10 variables. In general, there is a unique non-zero solution.

In practice, due to measurement noise, the matrix $R$ is always full rank, and a tolerance must be chosen to determine the numerical rank. In Section 8 we explore this issue through simulation.

\section{Implementation and Results}

In this section we present experimental results which validate the proposed reconstruction algorithm. A novel catadioptric mirror array is introduced and used for laboratory data collection. In addition, the sensitivity of the proposed method to calibration errors is explored via simulation.

\subsection{Spherical Catadioptric Mirror Array}

In order to experimentally confirm our proposed reconstruction algorithm, we designed and built a novel catadioptric imaging system consisting of an array of spherical mir- rors and a single, high-resolution perspective camera. A $1 / 4^{\prime \prime}$ thick anodized aluminum plate was used to hold 31 spherical mirrors within the field of view of an Olympus C80808 megapixel digital camera. Typical images acquired by this system are shown in Figures 1, 3, and 5 .

To obtain precise intrinsic and extrinsic model parameters, a three stage calibration procedure was used: (1) intrinsic calibration of the camera, (2) estimation of plate pose with respect to the camera, and (3) calibration of the spherical mirrors with respect to the plate. The last step includes estimating the location of the sphere centers, their radii, and the pose of a calibration object using an iterative bundleadjustment algorithm. The construction of our array, its calibration, and additional experimental results are presented in [13].

\subsection{Single Image Reconstruction Results}

In this section we discuss the implementation of our single image 3D line reconstruction algorithm using the spherical mirror array. First, as shown in Figure 3, we manually select four collinear points in a single image of the planar checkerboard pattern. A sub-pixel corner detection algorithm [2] is used to refine the manually-selected corners. Following the method outlined in Sections 5 and 6, we obtain an estimate of the equation of the 3D line.

A typical reconstruction is shown in Figure 3. Since ground truth is not available, we compare the single mirror reconstruction (shown in red) to that obtained using all 31 mirrors (shown in green). For this example, we find a translational error of $0.29 \mathrm{~mm}$ and an orientation error of $32.0^{\circ}$. As will be explained in Section 8.3, the accuracy of our reconstruction is currently limited by the calibration and physical construction of the mirror array and digital camera. We will determine the necessary calibration precision, by simulation, in the following section.

\subsection{Calibration Requirements}

As presented in [13], the proposed calibration algorithm is sufficient for reconstruction using multiple mirrors, however reconstruction from a single mirror requires higher precision. For the previous example, we believe reconstruction errors stem from three key limitations of our current system: (1) the distortion model used for the camera, (2) possible asphericity of the mirrors, and (3) limitations of the current calibration procedure for single mirror reconstruction. In order to guide the design of a future experimental system, we explore these issues via simulation.

To evaluate the necessary calibration accuracy for our proposed algorithm, we simulate the effect of random perturbations of the reflected rays $v$ on the reconstruction. The mean radius of the errors is determined by measuring the average distance of the reflected rays $v$ (cyan lines) from the 
best-estimate line (green line) as shown in Figure 3. Experimentally, we find that the estimated reflected rays $v$ found in the previous example do not approach closer than $0.4 \mathrm{~mm}$ (on average) to the best-estimate line.

As shown in Figure 4 we add random perturbations (distributed on a sphere of radius $0.4 \mathrm{~mm}$ ) to the bestestimate checkerboard positions. Using the experimentallydetermined points of intersection with the optical axis $w$, we construct the set of ideal reflected rays $v$. Applying our SVD-based reconstruction algorithm, we obtain an estimate of the equation of the intersecting line (shown in red). Note that this result is similar to that obtained experimentally.

In general, improvements in calibration should cause the reflected rays to pass closer to the best-estimate line. In simulation, we consider this effect by simply decreasing the perturbation radius until we achieve a reliable reconstruction of the checkerboard line. As shown in Figure 4 a perturbation radius of $4 \mu \mathrm{m}$ leads to reliable reconstructions. For this example, the translation error was $59 \mu \mathrm{m}$ and the orientation error was $0.51^{\circ}$.

\subsection{Optical Axis Baseline Requirements}

As discussed in Section 7, there exist classes of 3D lines which cannot be reconstructed by our system, regardless of the calibration precision. Experimentally, we find that calibration errors and certain viewing geometries result in nearly-coplanar lines - preventing a unique reconstruction.

As shown in Figure 3, the reflected rays $v$, when extended backwards, intersect the optical axis $w$ at four unique points (as discussed in Section 3). This is demonstrated graphically in the figure by the intersection of the yellow backwards viewing rays with the black optical axis. Note that, for this example, the intersections along the optical axis are nearly coincident - resulting in a small optical axis "baseline".

For this example, we find that the per-point average error of $0.4 \mathrm{~mm}$ is comparable to the optical axis baseline, measured to be $2.9 \mathrm{~mm}$. In this situation, the four viewing rays $v$ effectively intersect in a single point along the optical axis $w$. As a result, a correct reconstruction of the line is prevented, since, numerically, the four viewing rays $v$ are coplanar.

From this example we can conclude that calibration requirements are coupled to the specific viewing geometry; larger optical axis baselines will allow larger measurement errors, since the reflected rays will remain numerically noncoplanar and, as a result, allow a unique reconstruction.

\subsection{Reconstruction using Two Mirrors}

Although the focus of this paper is on reconstructing a 3D line using a single catadioptric image, we verify our experimental configuration by reconstructing a line using two

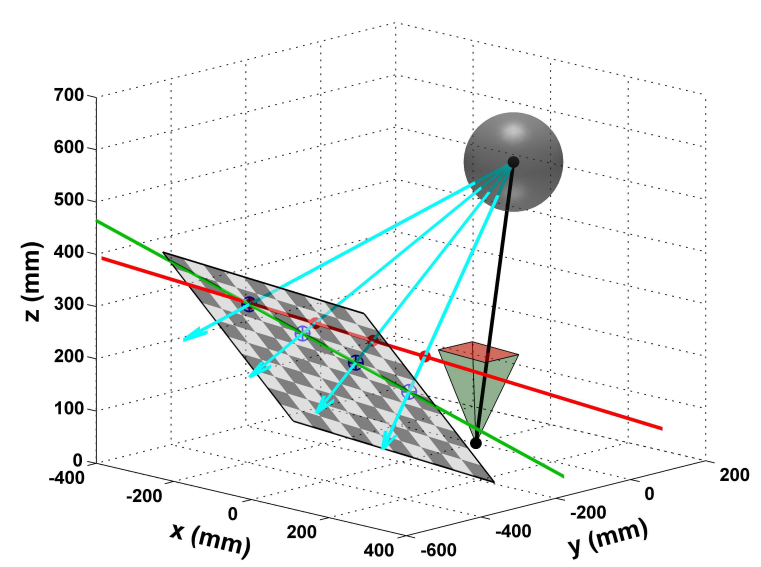

(a) Simulation results for $0.4 \mathrm{~mm}$ error radius.

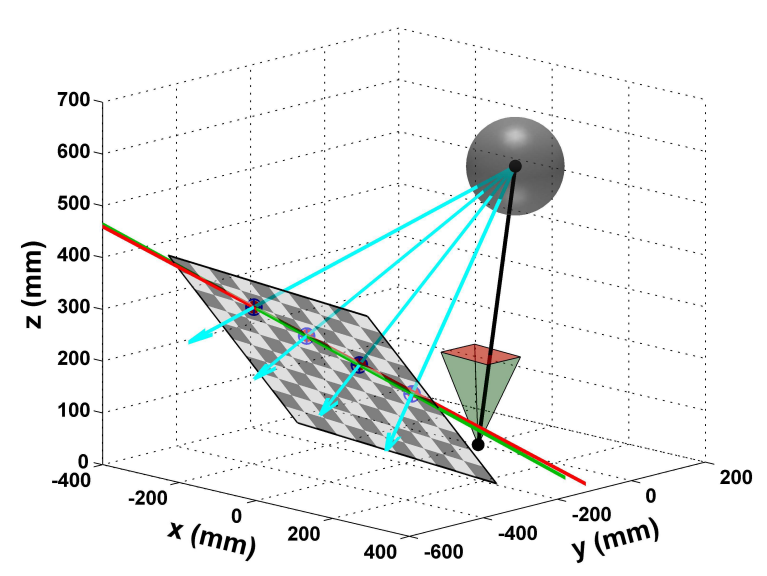

(b) Simulation results for $4 \mu \mathrm{m}$ error radius.

Figure 4: Simulation results for calibration sensitivity.

mirrors. This approach is similar to that presented by Nayar [14]. As shown in Figure 5, we manually select four points, in a neighboring mirror, that correspond to those used in the previous example. These image points are used to estimate the reflected rays $v$ and the point of intersection $q$ with each sphere. We use each corresponding pair of rays to triangulate the $3 \mathrm{D}$ position of each point on the line. Afterward, a line is fit to these points using linear least-squares (shown in red in the figure). For this specific example, we find that the resulting estimate deviates in translation by only $54 \mu \mathrm{m}$ and in orientation by $3.1^{\circ}$. This confirms that, given a sufficient baseline, our array can be used successfully for 3D point reconstruction - highlighting the greater calibration requirements necessitated by the proposed single image method.

\section{Conclusion}

In this paper we have shown that the equation of a $3 \mathrm{D}$ line can be estimated using only four points extracted from 


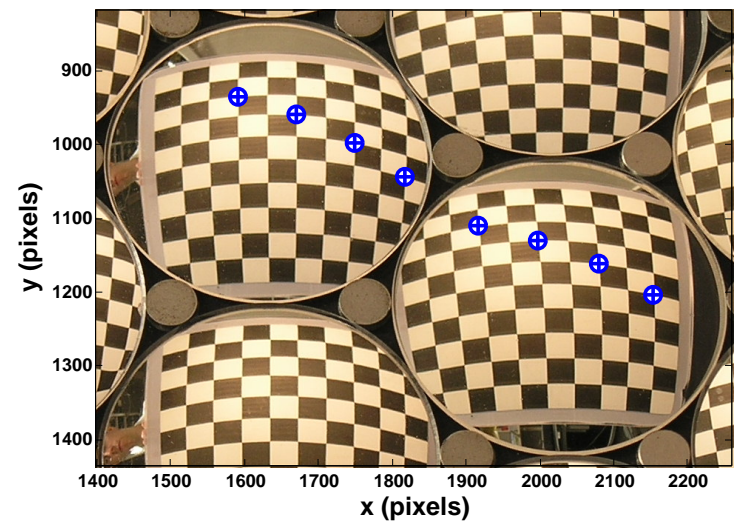

(a) Two neighboring mirrors and the points used for reconstruction.

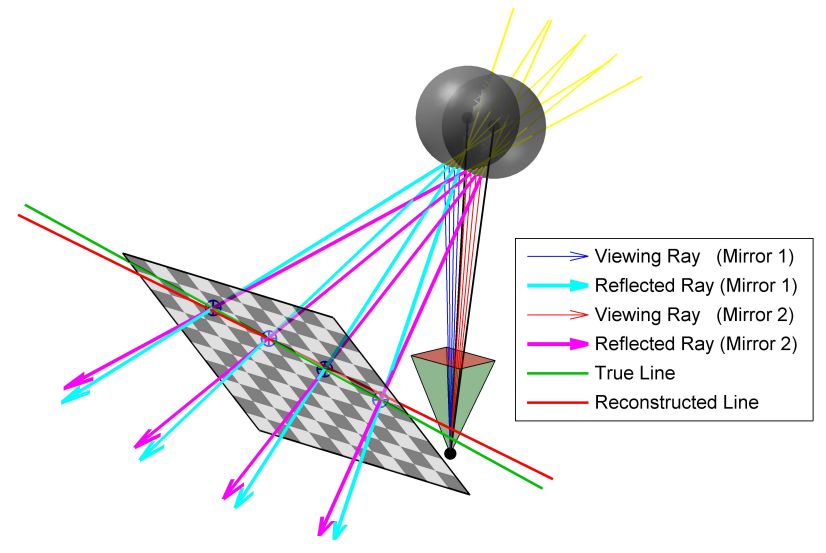

(b) $3 \mathrm{D}$ reconstruction of labeled points.

Figure 5: 3D reconstruction using two catadioptric images. The mirrors shown in (a) are represented by gray spheres in (b). The checkerboard is placed at its optimized position.

a single image acquired with an axial non-central optical system. A general algorithm based on computing the Singular Value Decomposition of the matrix of Plücker coordinates was presented. Preliminary experiments and simulations validate the proposed method and highlight the necessity of high-precision calibration for reliable reconstruction. Future studies will focus on developing more robust acquisition platforms and calibration procedures.

\section{References}

[1] J. P. Barreto and H. Araujo. Geometric Properties of Central Catadioptric Line Images. In Procedings of the 7th European Conference on Computer Vision, May 2002.

[2] J.-Y. Bouguet. Camera Calibration Toolbox for Matlab. http: / /www.vision. caltech. edu/bouguet j.

[3] V. Caglioti and S. Gasparini. Localization of 3D lines from single images using off-axis catadioptric cameras. In $O M$ NIVIS, Bejing, China, Oct. 2005.
[4] B. Chazelle, H. Edelsbrunner, L. Guibas, and M. Sharir. Lines in space-combinators, algorithms and applications. In Proceedings of the 21st Annual ACM Symposium on Theory of Computing, pages 382-393, Seattle, Washington, 1989.

[5] F. Cukierman. Introducción al Cálculo de Schubert. Course Notes in Primer Encuentro Nacional de Algebra, Vaquerías, Cordoba, Argentina, Dec. 2003.

[6] C. Geyer and K. Daniilidis. Catadioptric Camera Calibration. In Proceedings 7th International Conference on Computer Vision, pages 398-404, 1999.

[7] C. Geyer and K. Daniilidis. Catadioptric projective geometry. International Journal of Computer Vision, 45(3):223243, 2001.

[8] C. Geyer and K. Daniilidis. Properties of the Catadoptric Fundamental Matrix. In Proceedings of the 7th European Conference on Computer Vision, Copenhagen, Denmark, 2002.

[9] M. Grossberg and S. Nayar. A General Imaging Model And A Method For Finding Its Parameters. In ICCV, 2001.

[10] R. A. Hicks and R. Perline. The Fixed Surface Method for Catadioptric Sensor Design. Technical report, Drexel University Department of Mathematics, 2004.

[11] S. Kang. Catadioptric Self-Calibration. In Proceedings of the IEEE Conference on Computer Vision and Pattern Recognition, pages 201-207, June 2000.

[12] S. L. Kleiman and D. Laksov. Schubert Calculus. American Mathematical Monthly, 79:1061-1082, Dec. 1972.

[13] D. Lanman, D. Crispell, M. Wachs, and G. Taubin. Spherical catadioptric arrays: Construction, multi-view geometry, and calibration. In Third International Symposium on 3D Data Processing, Visualization and Transmission, 2006.

[14] S. Nayar. Sphereo: Determining Depth using Two Specular Spheres and a Single Camera. In Proceedings of SPIE Conference on Optics, Illumination, and Image Sensing for Machine Vision III, pages 245-254, Nov. 1988.

[15] S. Nene and S. Nayar. Stereo Using Mirrors. In Proceedings of the International Conference on Computer Vision (ICCV'98), Bombay, India, 1998.

[16] H. Schubert. Kalkül der Abzählenden Geometrie. Teubner, Leipzig, 1874.

[17] J. Stolfi. Primitives for Computational Geometry. $\mathrm{PhD}$ thesis, Computer Science Department, Stanford University, 1988.

[18] J. Stolfi. Oriented Projective Geometry. Academic Press, San Diego, 1991.

[19] P. Sturm. Multi-View Geometry for General Camera Models. In $C V P R, 2005$.

[20] R. Swaminiathan, M. Grossbery, and S. Nayar. Caustics of Catadioptric Cameras. In Proceedings of the 8th International Conference on Computer Vision, July 2001.

[21] S. Teller and M. Hohmeyer. Determining the Lines through Four Lines. Journal of Graphics Tools, 4(3):11-22, 1999.

[22] X. Ying and Z. Hu. Catadioptric Camera Calibration Using Geometric Invariants. IEEE Transactions On Pattern Analysis and Machine Intelligence, 26(10), Oct. 2004. 